\title{
Inelastic electron-vortex-beam scattering
}

\author{
Ruben Van Boxem, ${ }^{*}$ Bart Partoens, and Jo Verbeeck \\ EMAT \& CMT, University of Antwerp, Groenenborgerlaan 171, 2020 Antwerpen, Belgium
}

(Received 3 February 2015; published 16 March 2015)

\begin{abstract}
Recent theoretical and experimental developments in the field of electron-vortex-beam physics have raised questions about what exactly this novelty in the field of electron microscopy (and other fields, such as particle physics) really provides. An important part of the answer to these questions lies in scattering theory. The present investigation explores various aspects of inelastic quantum scattering theory for cylindrically symmetric beams with orbital angular momentum. The model system of Coulomb scattering on a hydrogen atom provides the setting to address various open questions: How is momentum transferred? Do vortex beams selectively excite atoms, and how can one employ vortex beams to detect magnetic transitions? The analytical approach presented here provides answers to these questions. OAM transfer is possible, but not through selective excitation; rather, by pre- and postselection one can filter out the relevant contributions to a specific signal.
\end{abstract}

DOI: 10.1103/PhysRevA.91.032703

PACS number(s): 03.65.Nk, 03.65.Vf, 34.80.Dp

\section{INTRODUCTION}

The field of phase vortices in beams has a relatively short but vibrant history, beginning in optics and ending in all forms of wave mechanical systems. A good overview of theory and applications in optics can be found in Refs. [1] and [2]. Interesting aspects include the quantization of orbital angular momentum (OAM) [3], the optomechanical effects of these beams $[3,4]$, quantum information, computing, and communication uses [5,6], and, of course, scattering applications [7-9]. Various ways of obtaining electron vortex beams have been demonstrated [10-17] since their inception on paper in 2007 [18] and experimentally in 2010 [12,19]. Several investigations into the scattering aspects of electron vortex beams have been published [20,21], and this work aims to add to that list a thorough and general analysis of inelastic electron vortex scattering.

The main questions we investigate in this paper pertain to the detection of and mechanism of OAM transfer of a fast vortex electron to an atomic system. The results presented in this paper describe the result of a detailed investigation into the quantum dynamics of OAM transfer from an incoming electron vortex beam to an atomic electron. It is an attempt to describe what the incoming and outgoing OAM and transverse momentum do to the scattering amplitude and how these can be exploited in discovering what happened during a scattering event. In what follows, inelastic scattering of fast electron Bessel beams is calculated for the model system of a hydrogen atom, for which several transition amplitudes are explicitly calculated. Although we consider a spherically symmetric atomic system, we prefer to discuss everything relevant to the beam axis, and we treat transitions with OAM transfer in that view. Special attention is given to how and when selection rules are present, and if they are not, we describe the physical reasons behind their vanishing outside of special circumstances. We put the emphasis on what vortex beams contribute to scattering and what they do not. To this effect, two alternative approaches are pursued, allowing for a sufficiently nuanced and accurate report on the properties

\footnotetext{
*ruben.vanboxem@uantwerpen.be
}

of electron-vortex-beam atomic excitations. Critical are the concepts of pre- and postselection on the scattered wave, and special attention is given to transitions in which the beam's OAM is transfered to the atomic electron. Effects related to relativity, spin, or recoil of the nucleus are ignored. This treatment is also more simplistic than the more advanced treatments based on the density matrix [22,23] and relate more closely to earlier theoretical advances in inelastic electronatom scattering [24-26].

The next section quickly reviews various inelastic scattering concepts and puts special emphasis on the details often omitted in a textbook treatment of quantum scattering on atomic systems, which are important here. Section III presents the two methods developed to attack this problem analytically. Section IV is a discussion of the most important results. The final section presents our conclusions.

\section{Notation}

In order to remove clutter from crucial steps in the calculation, strict notational conventions are defined here. Real-space coordinates are denoted $\boldsymbol{r}=(x, y, z)=\left(r_{\perp}, \varphi, z\right)$. Basis vectors are written as $\boldsymbol{e}_{i}$, where $i$ is the relevant coordinate. Momentum-space coordinates are denoted $\boldsymbol{k}=$ $\left(k_{x}, k_{y}, k_{z}\right)=\left(k_{\perp}, \phi, k_{z}\right)$. It is often advantageous to split any three-dimensional (3D) vector $\boldsymbol{v}$ into a $z$ component, $v_{z}$, and a perpendicular component, $\boldsymbol{v}_{\perp}=\left(v_{x}, v_{y}\right)$, with size $v_{\perp}$. Primed variables are denoted $\boldsymbol{r}^{\prime}=\left(x^{\prime}, y^{\prime}, z^{\prime}\right)=\left(r_{\perp}^{\prime}, \varphi^{\prime}, z^{\prime}\right)$, and similarly for $\boldsymbol{k}^{\prime}$. Partial derivatives are denoted $\partial_{x}=\frac{\partial}{\partial x}$ and are taken to act on everything to the right of the symbol. Primed coordinates and quantum numbers refer to the final (outgoing) state. Momentum transfer is denoted $\boldsymbol{q}$ and is equal to $\boldsymbol{k}-\boldsymbol{k}^{\prime}$. OAM transfer is denoted $\Delta m$ and is equal to $m-m^{\prime}$.

\section{A CYLINDRICAL VIEW OF INELASTIC ELECTRON PLANE-WAVE SCATTERING}

\section{A. Inelastic scattering of an electron on an atom}

In this section, various entities are defined and plane-wave scattering theory is briefly reviewed. Certain often forgotten aspects which are important to the cylindrical-wave case are brought forward. 


\section{Interaction potential}

The interaction potential for an electron scattering on a single-electron atomic system is given by

$$
V=e^{2}\left(\frac{1}{\left|\boldsymbol{r}-\boldsymbol{r}^{\prime}\right|}-\frac{Z}{r^{\prime}}\right) \text {. }
$$

Here, $\boldsymbol{r}^{\prime}$ is the laboratory coordinate for the scattering electron with respect to the nucleus, and $\boldsymbol{r}$ is the atomic electron's coordinate relative to the nucleus (which is located at the origin). Extending this to multiple single-electron atomic states is formally trivial: summing over every electron's coordinate suffices, so that $\boldsymbol{r} \rightarrow \sum \boldsymbol{r}_{j}$. The relative distance $\left|\boldsymbol{r}-\boldsymbol{r}^{\prime}\right|$ complicates the cylindrical scattering treatment in the next sections.

\section{Single-electron atomic wave functions}

To clarify the role of the atomic system's OAM, we assume that the (projected) OAM is a good quantum number and separate the atomic wave functions into an azimuthal part and the remaining $\left(r_{\perp}, z\right)$ part:

$$
|i\rangle=\frac{e^{i m \varphi}}{\sqrt{2 \pi}}|\alpha\rangle, \quad|f\rangle=\frac{e^{i m^{\prime} \varphi}}{\sqrt{2 \pi}}|\beta\rangle .
$$

For a hydrogen-like atom with (effective) nuclear charge $Z$ and atomic radius $a_{\mu}=a_{0} m_{e} / \mu$ ( $\mu$ is the reduced mass of the atomic system), orthonormal wave functions have the form

$$
\langle\boldsymbol{r} \mid n l m\rangle=\sqrt{\eta^{3} \frac{(n-l-1) !}{2 n(n+1) !}} \frac{e^{i m \varphi}}{\sqrt{2 \pi}}(\eta r)^{l} L_{n-l-1}^{2 l+1}(\eta r) e^{-\frac{\eta r}{2}},
$$

where $\eta=2 Z /\left(n a_{\mu}\right)$, and $n=1,2,3 \ldots, l=0, \ldots n-1$, and $m=-l, \ldots l$ are the radial, angular, and magnetic quantum numbers, respectively. The OAM of a state, determined by the operator $\hat{L}$, is given by $l$, and the projected OAM, given by $\hat{L}_{z}$ and the one of primary interest here, is given by $m$. In what follows, we assume that $\mu \approx m_{e}$ and so $a_{\mu} \approx a_{0}$ but keep $Z$ so that any dependence on atomic number is clear.

\section{Scattering amplitude}

The inelastic scattering amplitude in the first Born approximation can be written as

$$
f_{f i}\left[\boldsymbol{k}^{\prime}, \Phi\right]=-\frac{2 m_{e} N}{4 \pi \hbar^{2}}\left\langle\boldsymbol{k}^{\prime}|\langle f|V| i\rangle| \Phi\right\rangle,
$$

where $N$ takes care of the normalization of the scattering electron's states [27], $|i\rangle$ and $|f\rangle$ are the initial and final atomic states, and $|\Phi\rangle$ is the incoming state (traditionally taken to be a plane wave). The outgoing plane wave is defined as

$$
\left\langle\boldsymbol{r} \mid \boldsymbol{k}^{\prime}\right\rangle=\frac{e^{i \boldsymbol{k}^{\prime} \cdot \boldsymbol{r}}}{(2 \pi)^{3 / 2}} .
$$

The scattering amplitude determines the modulation of the outgoing (scattered) spherical wave so that the total wave function obeys the following relation:

$$
\Psi(\boldsymbol{r})=\Phi(\boldsymbol{r})+f_{f i}\left[\boldsymbol{k}^{\prime}, \Phi\right] \frac{e^{i \boldsymbol{k} \cdot \boldsymbol{r}}}{r} .
$$

The scattering amplitude, Eq. (4), depends on the atomic states involved in a certain transition, and thus the probability of scattering an electron in a certain direction depends on these states.
Filling in Eq. (4) with Eq. (1), we obtain

$$
\begin{aligned}
& f_{f i}^{\mathrm{B}}\left[\boldsymbol{k}^{\prime}, \Phi\right] \\
& \quad=-\frac{m_{e} e^{2} N}{2 \pi \hbar^{2}}\left(\left\langle\boldsymbol{k}^{\prime}\left|\left\langle f\left|\frac{1}{\left|\boldsymbol{r}-\boldsymbol{r}^{\prime}\right|}\right| i\right\rangle\right| \Phi\right\rangle-Z\langle f \mid i\rangle\left\langle\boldsymbol{k}^{\prime}\left|\frac{1}{r^{\prime}}\right| \Phi\right\rangle\right) .
\end{aligned}
$$

\section{The final state}

The outgoing electron's momentum $k^{\prime}$ is related to the incoming electron's momentum $k$ through the energy transferred to the atomic system $\Delta E$ as follows:

$$
k^{\prime 2}=k^{2}-\frac{2 m_{e}}{\hbar^{2}} \Delta E .
$$

The scattering angle $\theta$ can be introduced by substituting

$$
q^{2}=k^{2}+k^{\prime 2}-2 k k^{\prime} \cos \theta .
$$

Using the above with Eq. (7) and Eq. (3), one can plot the angular dependence of the scattering amplitudes for various hydrogen transitions. These are shown in Fig. 1(a). The different final states $(s / p / d / \ldots)$ result in specific angular regions in which some dominate or are suppressed with respect to others, which implies that some transitions can be filtered out roughly by postselection on $\theta$ (which directly corresponds to the outgoing transverse momentum). This can be exploited to map anisotropic bonding in crystals [28,29].

We now consider a ground-state excitation to a state with fixed $\left(n^{\prime}, l^{\prime}\right)$, e.g., the $2 p$ orbitals, and see if the outgoing wave's OAM can be used to provide more details of the final state. The question we ask ourselves is: What determines scattering to a particular (projected) OAM? This example final state consists of three substates: $2 p_{z}, 2 p_{+}$, and $2 p_{-}$. The orientation of these final states is determined by the direction of the $z^{\prime}$ axis, along which their angular momentum is projected to give the quantum number $m^{\prime}$. This is crucial to the whole analysis. Using the Fourier translation theorem, the plane-wave scattering amplitude becomes

$$
f_{f i}^{\mathrm{PW}}(\boldsymbol{q})=-\frac{2 m_{e} e^{2}}{\hbar^{2}}\left\langle f\left|\frac{e^{i \boldsymbol{q} \cdot r^{\prime}}-Z}{q^{2}}\right| i\right\rangle .
$$

This can be calculated in position space by aligning the $z^{\prime}$ axis along the momentum transfer vector $\boldsymbol{q}$ :

$$
f_{f i}^{\mathrm{PW}}(\boldsymbol{q})=-\frac{2 m_{e} e^{2}}{\hbar^{2}} \int \mathrm{d}^{3} \boldsymbol{r}^{\prime} \psi_{f}^{*}\left(\boldsymbol{r}^{\prime}\right) \frac{e^{i q r^{\prime} \cos \theta^{\prime}}-Z}{q^{2}} \psi_{i}\left(\boldsymbol{r}^{\prime}\right) .
$$

The final states are thus automatically quantized along the direction of $\boldsymbol{q}$, when expressed in the $\boldsymbol{r}^{\prime}$ coordinate system [30]. Furthermore, due to the integration over the azimuthal coordinate $\varphi^{\prime}$ in Eq. (11), only the tilted states for which $\Delta m=$ 0 contribute. In our example of ground-state excitation, this means that for a certain $\left(n^{\prime}, l^{\prime}\right)$, only the state with $m^{\prime}=0$ gives a nonzero contribution. Indeed, the transition amplitude for $1 s \rightarrow 2 p$ is given by the following expression:

$$
\chi, \phi^{\prime}\left\langle 2 p_{z}\left|\frac{e^{i \boldsymbol{q} \cdot \boldsymbol{r}}}{q^{2}}\right| 1 s\right\rangle=\left(\frac{Z}{a_{0}}\right)^{5} \frac{12 i \sqrt{2}}{q\left[q^{2}+\left(\frac{3 Z}{2 a_{0}}\right)^{2}\right]^{3}} .
$$



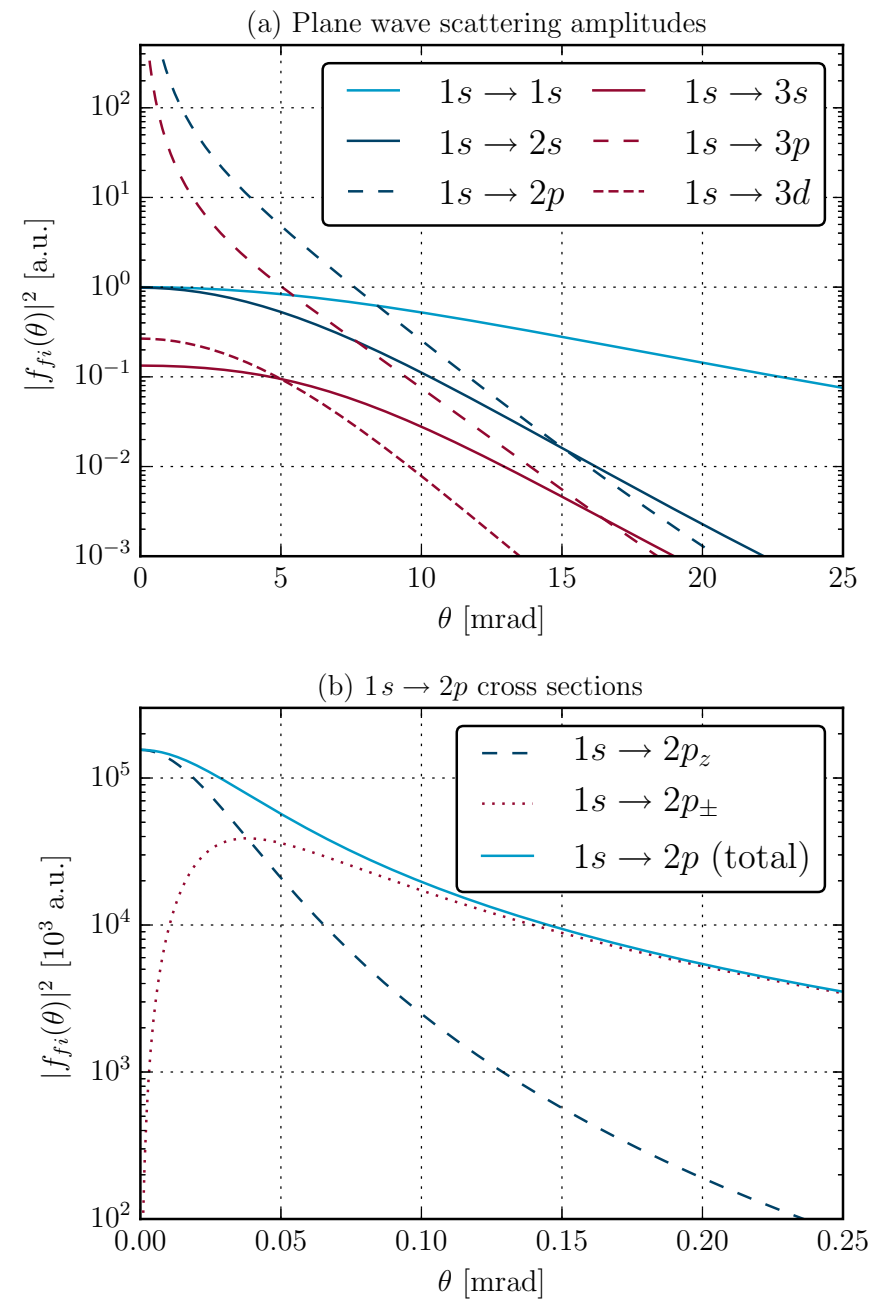

FIG. 1. (Color online) (a) Plane-wave scattering amplitudes for $120-\mathrm{keV}$ electrons for various transitions from the ground state. One can see the relative size of the amplitudes shifts depending on the exact scattering angle in question. (b) Separate cross sections for $2 p_{z}$ and $2 p_{ \pm}$excitation. The typical Lorentz profile appears [28]. The total $1 s \rightarrow 2 p$ cross section is the sum of the other three. Note that the $\theta$ scale in (b) is a factor of 100 times smaller than the one in (a). This tiny scale is due to the relatively small $\Delta E$ for pure hydrogen. More realistic systems will show the same features at a much larger scale.

The subscript $\chi$ expresses that the quantization axis is tilted over an angle of $\chi$, defined by

$$
\tan \chi=\frac{q_{\perp}}{q_{z}}=\frac{k^{\prime} \sin \theta}{k-k^{\prime} \cos \theta},
$$

and rotated around the beam's $z$ axis by an angle $\phi^{\prime}$. This scattering geometry is shown in Fig. 2. The reader can verify that Eq. (12) is 0 for the other tilted substates (in our example, these are $2 p_{+}$and $\left.2 p_{-}\right)$. This tilted state $\left(z^{\prime}\right.$ is rotated with respect to the beam's $z$ axis) can be projected onto untilted states which are quantized along the beam direction.

As the atomic orbitals are determined by a radial function multiplied with an OAM eigenstate, $|l, m\rangle$, their rotation properties are fully determined by the Wigner $d$ matrix:

$$
\left\langle l, m|\hat{R}(\alpha, \beta, \gamma)| l^{\prime}, m^{\prime}\right\rangle=\delta_{l^{\prime}, l} D_{m, m^{\prime}}^{(l)}(\alpha, \beta, \gamma) .
$$

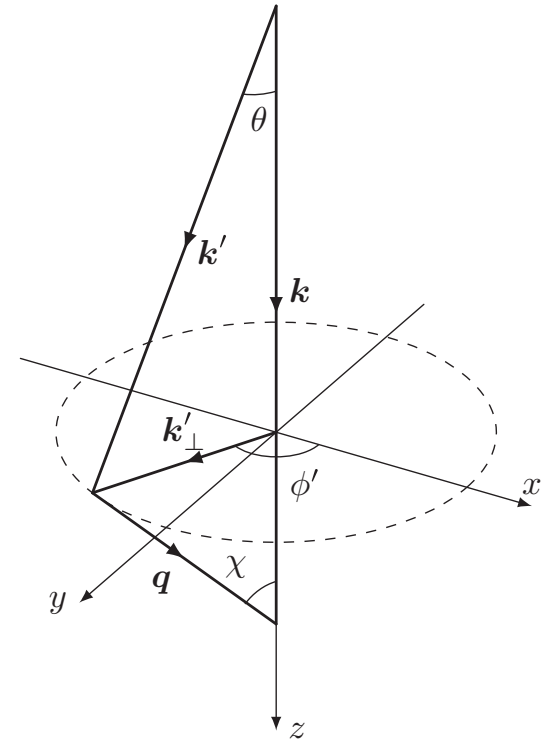

FIG. 2. For plane-wave scattering, the orientation of the final state depends on the scattering angle $\theta$ and the transition energy $\Delta E$ as shown in Eq. (13). The azimuthal angle $\phi^{\prime}$ of the outgoing wave with respect to a fixed set of axes is also shown.

Rotating an angular momentum eigenstate over the angles $\alpha$, $\beta$, and $\gamma$ (in the $z-y-z$ convention) turns it into a sum of states of equal $l$, but all $m$ :

$$
\hat{R}(\alpha, \beta, \gamma)\left|l, m^{\prime}\right\rangle=\sum_{m=-l}^{+l} D_{m, m^{\prime}}^{(l)}(\alpha, \beta, \gamma)|l, m\rangle .
$$

For the rotation of an $m^{\prime}=0$ state oriented along the vector $\boldsymbol{q}$ as in Fig. 2, the matrix elements take on the form

$$
\begin{aligned}
D_{m, 0}^{(l)}\left(\phi^{\prime}, \chi, 0\right) & =e^{-i m \phi^{\prime}} d_{m, 0}^{(l)}(\chi) \\
& =e^{-i m \phi^{\prime}} \sqrt{\frac{(l-m) !}{(l+m) !}} P_{l}^{m}(\cos \chi),
\end{aligned}
$$

where $P_{l}^{m}$ are the associated Legendre polynomials.

As an example, consider the $1 s \rightarrow 2 p$ transitions of the hydrogen atom, which can readily be calculated by filling in Eq. (11) with Eq. (3). With respect to the beam direction, the excited $2 p_{z}$ state is tilted by the angle $\chi$ as shown in Fig. 2. Applying Eqs. (15) and (16), for this example, one obtains

$$
\left|2 p_{z}\right\rangle_{\chi, \phi^{\prime}}=\frac{\sin \chi}{\sqrt{2}}\left(e^{i \phi^{\prime}}\left|2 p_{-}\right\rangle-e^{-i \phi^{\prime}}\left|2 p_{+}\right\rangle\right)+\cos \chi\left|2 p_{z}\right\rangle \text {. }
$$

Here,

$$
\sin \chi=\frac{q_{\perp}}{q}, \quad \cos \chi=\frac{q_{z}}{q} .
$$

This expression gives the projection of the tilted state $\left|2 p_{z}\right\rangle_{\chi, \phi^{\prime}}$ into its untilted components (quantized with respect to the beam's $z$ direction).

The explicit form of the projection of the $\boldsymbol{q}$-oriented $2 p_{z}$ state onto a beam-axis-oriented $2 p$ state, e.g., $\left\langle 2 p_{ \pm} \mid 2 p_{z}\right\rangle_{\chi, \phi^{\prime}}$, in Eq. (17), allows us to write the scattering amplitude to, e.g., 
the $2 p_{ \pm}$states directly using Eq. (12):

$$
\begin{aligned}
f_{1 s \rightarrow 2 p_{ \pm}}^{\mathrm{PW}} & =-\frac{2 m_{e} e^{2}}{\hbar^{2}}\left\langle 2 p_{ \pm}\left|\frac{e^{i \boldsymbol{q} \cdot \boldsymbol{r}}}{q^{2}}\right| 1 s\right\rangle \\
& =-\frac{2 m_{e} e^{2}}{\hbar^{2}}\left\langle 2 p_{ \pm} \mid 2 p_{z}\right\rangle_{\chi, \phi^{\prime} \chi, \phi^{\prime}}\left\langle 2 p_{z}\left|\frac{e^{i \boldsymbol{q} \cdot \boldsymbol{r}}}{q^{2}}\right| 1 s\right\rangle \\
& =\left(\frac{Z}{a_{0}}\right)^{5} e^{\mp i \phi^{\prime}} \frac{-12 i m_{e} e^{2} q_{\perp}}{\hbar^{2} q^{2}\left[q^{2}+\left(\frac{3 Z}{2 a_{0}}\right)^{2}\right]^{3}} .
\end{aligned}
$$

This is obtained by inserting a complete $l=1$ basis and remembering that the transition element is only nonzero for $\left|2 p_{z}\right\rangle_{\chi, \phi^{\prime}}$, as discussed above. Note that the outgoing wave has acquired the opposite OAM of the excited atomic state, and thus OAM has been transferred to the bound electron. The above result is not new, but it usually appears as a cross section. More specific forms are exploited in, e.g., momentum-resolved EELS experiments [31,32], where dynamical diffraction also plays a large role in the final distribution of scattered electrons. The $2 p_{z}$ excitation, where no OAM is transferred, can be calculated directly as well:

$$
f_{1 s \rightarrow 2 p_{z}}^{\mathrm{PW}}=\left(\frac{Z}{a_{0}}\right)^{5} \frac{-12 \sqrt{2} i m_{e} e^{2} q_{z}}{\hbar^{2} q^{2}\left[q^{2}+\left(\frac{3 Z}{2 a_{0}}\right)^{2}\right]^{3}} .
$$

Both Eq. (18) and Eq. (19) are shown in Fig. 1(b). These scattering amplitudes can be separately observed if one filters the outgoing wave by its OAM. Techniques to achieve this exist for electromagnetic waves [33,34], but in electron optics these are still in development $[35,36]$.

\section{B. The vortex-beam basis state: Bessel beams}

The simplest form of vortex beams is provided by the solution of the Schrödinger equation in cylindrical coordinates:

$$
\langle\boldsymbol{r} \mid \boldsymbol{k}, \ell\rangle=\psi_{\boldsymbol{k}, \ell}(\boldsymbol{r})=\frac{e^{i \ell \varphi}}{\sqrt{2 \pi}} J_{\ell}\left(k_{\perp} r_{\perp}\right) \frac{e^{i k_{z} z}}{\sqrt{2 \pi}} .
$$

This exact solution encompasses the beam features that interest us: the quantized (projected) OAM $\hbar \ell$ and the longitudinal and transverse momenta $\hbar k_{z}$ and $\hbar k_{\perp}$. One can define the opening angle as $\alpha=\tan ^{-1}\left(k_{\perp} / k_{z}\right)$, which is the angle the momentum vector makes with the $z$ axis. The energy of this field-free state is independent of its OAM: $E=\hbar^{2}\left(k_{z}^{2}+k_{\perp}^{2}\right) /\left(2 m_{e}\right)$. The Bessel beam can be written in terms of its momentum components, which shows that this state is a ring of tilted plane waves in momentum space:

$$
\psi_{\boldsymbol{k}, \ell}(\boldsymbol{r})=(-i)^{\ell} \int \frac{\mathrm{d} \phi}{(2 \pi)^{2}} e^{i \ell \phi} e^{i \boldsymbol{k} \cdot \boldsymbol{r}} .
$$

This representation was used to calculate the elastic Coulomb scattering amplitude in an earlier work [27], the results of which are useful here in simplifying some of the equations.

Bessel beams are basis states, much like plane waves. It is impossible to create true Bessel beams, even in a laboratory setting. Only approximations can be realized, which admittedly show the various properties of real Bessel beams in a limited way [37]. Nonetheless, they provide a good basis to calculate scattering amplitudes because they encode features such as cylindrical symmetry, convergence angle (transverse momentum), and OAM in a natural way. Note that for a convergent beam, the wave function of the probe in real space is nothing more than a coherent superposition of Bessel-beam basis states, integrated over the aperture radius. For a nonvortex probe, this reads

$$
\Psi(\boldsymbol{r}) \propto \int_{0}^{\infty} \mathrm{d} k_{\perp} A\left(k_{\perp}\right) k_{\perp} J_{0}\left(k_{\perp} r_{\perp}\right) .
$$

Here, $A\left(k_{\perp}\right)$ describes the aperture (for example, as a step function). This is nothing new and is often called the Hankel transform. What this means is that the scattering amplitude of a real convergent beam can be obtained from that of a Bessel beam by integrating over a $k_{\perp}$ range given by the aperture radius. For a vortex probe, one can write

$$
\Psi_{\ell}(\boldsymbol{r}) \propto e^{i \ell \varphi} \int_{0}^{\infty} \mathrm{d} k_{\perp} A\left(k_{\perp}\right) k_{\perp} J_{\ell}\left(k_{\perp} r_{\perp}\right),
$$

which is nothing more than the $\ell$ th-order Hankel transform [38].

\section{INELASTIC VORTEX-BEAM SCATTERING}

\section{A. General formulation}

We consider a perfectly centered vortex, so that the atom lies exactly on the beam's OAM axis. Beam displacement can be taken into account by using the Bessel addition formula [see Eq. (25)] for the incoming beam in the transverse plane. Any nonzero displacement of the beam (which is to be expected in any realistic situation) will thus introduce a progressively larger number of OAM modes contributing significantly to the scattering. The extra modes contribute by a factor determined by the transverse momentum, displacement, and the OAM of that contribution through $J_{\ell-\mu}\left(k_{\perp} r_{0 \perp}\right)$, where $\ell$ is the pure OAM mode displaced over a distance $r_{0 \perp}$, and $\mu$ is the OAM of a mode introduced by the displacement. For $r_{0 \perp}=0$, all other contributions disappear. These additional OAM modes will coherently contribute to the final scattering amplitude and, thus, interfere with calculation of the differential cross section.

Following up on Ref. [27], one can calculate inelastic scattering amplitudes by replacing $|\Phi\rangle$ in Eq. (7) with a Bessel beam $|\boldsymbol{k}, \ell\rangle$, Eq. (20). Ignoring the (purely elastic) term (which was calculated in Ref. [27] and repeated in the Appendix), this leads to

$$
\begin{aligned}
f_{f i}^{\mathrm{V}}= & -\frac{m_{e} e^{2}}{2 \pi \hbar^{2}} \int \mathrm{d}^{3} \boldsymbol{r} \psi_{f}^{*}(\boldsymbol{r}) \psi_{i}(\boldsymbol{r}) \int \mathrm{d}^{3} \boldsymbol{r}^{\prime} e^{-i \boldsymbol{k}^{\prime} \boldsymbol{r}^{\prime}} \\
& \times \frac{1}{\left|\boldsymbol{r}-\boldsymbol{r}^{\prime}\right|} e^{i \ell \varphi^{\prime}} J_{\ell}\left(k_{\perp} r_{\perp}^{\prime}\right) e^{i k_{z} z} .
\end{aligned}
$$

The relative atomic coordinate of the atomic electron, $\boldsymbol{r}^{R}=\boldsymbol{r}-\boldsymbol{r}^{\prime}$, plays a central role in how to solve this problem. We would like to substitute this in the inner integral so as not to involve the atomic wave functions already. The relative distance $\left|\boldsymbol{r}-\boldsymbol{r}^{\prime}\right|$ is difficult to substitute directly in this expression, due to the presence of the transverse coordinate. There are two ways to proceed: use the Bessel addition theorem to mathematically displace the Bessel beam to this relative coordinate or introduce the Fourier representation of the Bessel beam, Eq. (21). Both methods lead to interesting physical insights, and thus both are treated in detail below. 


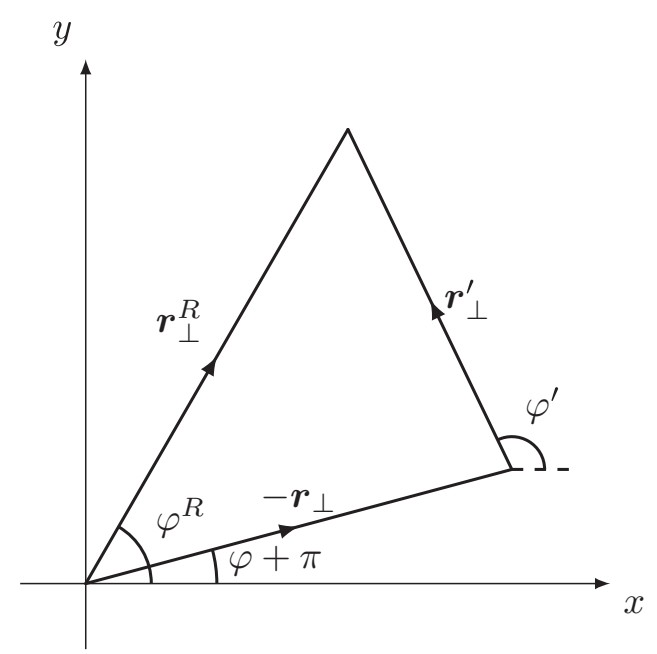

FIG. 3. In-plane geometry as used in Eq. (25) for the shift to the atomic electron's coordinate.

Note that much like the elastic results presented in a previous article [27], any of these scattering amplitudes can be summed or integrated over a certain range of $k_{\perp}, k_{z}$, and/or $\theta$ to more closely resemble real wave packets. This allows for modeling of a focused electron probe with a specific convergence angle (including annular apertures), or a certain collector angle range for comparison with the experiment.

\section{B. Displaced Bessel-beam representation}

The relative coordinate problem described above can be worked around by applying the Bessel addition theorem, by which a displaced Bessel beam can be replaced with a series of weighted centered Bessel functions over all orders. In our case, the theorem states [39]

$$
\begin{aligned}
& \frac{e^{i k_{z} z^{\prime}}}{\sqrt{2 \pi}} J_{\ell}\left(k_{\perp} r_{\perp}^{\prime}\right) \frac{e^{i \ell \varphi^{\prime}}}{\sqrt{2 \pi}} \\
& \quad=e^{i k_{z} z} \sum_{\mu=-\infty}^{+\infty} J_{\mu}\left(k_{\perp} r_{\perp}\right) e^{i \mu \varphi} \frac{e^{i k_{z} z^{R}}}{\sqrt{2 \pi}} J_{\ell-\mu}\left(k_{\perp} r_{\perp}^{R}\right) \frac{e^{i(\ell-\mu) \varphi^{R}}}{\sqrt{2 \pi}},
\end{aligned}
$$

where the relative coordinate is denoted $\boldsymbol{r}^{R}=\boldsymbol{r}-\boldsymbol{r}^{\prime}$. This is illustrated in Fig. 3.

Applying this to Eq. (24), one obtains

$$
\begin{aligned}
& f_{f i}^{\mathrm{V}}\left(\boldsymbol{k}^{\prime} ; \boldsymbol{k}, \ell\right)=-\frac{m_{e} e^{2} N}{2 \pi \hbar^{2}} \sum_{\mu=-\infty}^{+\infty}\left\langle f\left|e^{-i \boldsymbol{k}^{\prime} \cdot \boldsymbol{r}} J_{\mu}\left(k_{\perp} r_{\perp}\right) e^{i \mu \varphi}\right| i\right\rangle \\
& \times\left\langle\boldsymbol{k}^{\prime}\left|\frac{1}{r^{R}}\right| \boldsymbol{k}, \ell-\mu\right\rangle \\
& =-\sum_{\mu=-\infty}^{+\infty} f_{\mathrm{el}}^{V}\left(\boldsymbol{k}^{\prime} ; \boldsymbol{k}, \ell-\mu\right) \\
& \times\left\langle f\left|e^{-i \boldsymbol{k}_{\perp}^{\prime} \cdot \boldsymbol{r}_{\perp}} J_{\mu}\left(k_{\perp} \boldsymbol{r}_{\perp}\right) e^{i \mu \varphi} e^{i q_{z} z}\right| i\right\rangle \\
& =-\sum_{\mu=-\infty}^{+\infty} f_{\mathrm{el}}^{V}\left(\boldsymbol{k}^{\prime} ; \boldsymbol{k}, \ell-\mu\right) \widetilde{\mathcal{M}}_{f i}\left(\boldsymbol{k}^{\prime}, \boldsymbol{k}, \mu\right) \text {. }
\end{aligned}
$$

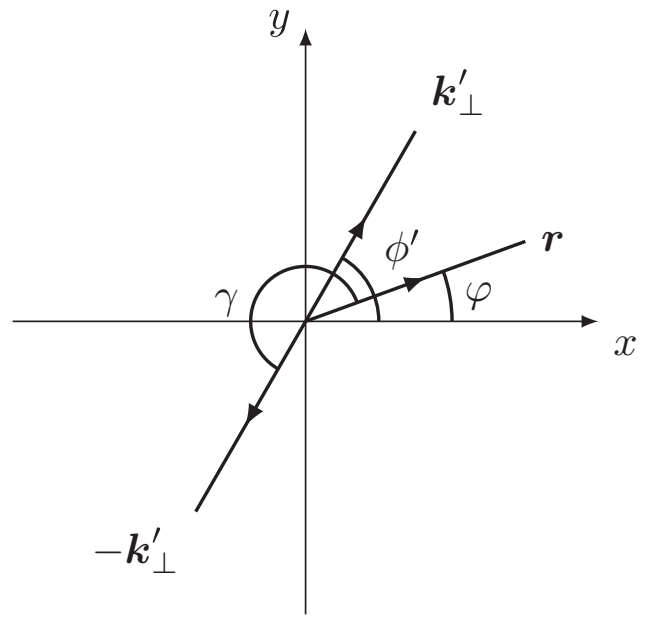

FIG. 4. Geometry for the outgoing plane wave's Jacobi-Anger expansion in Eq. (27). Here, $\gamma$ is the angle between $-\boldsymbol{k}_{\perp}$ and $\boldsymbol{r}_{\perp}$.

In the above expressions, $f_{\mathrm{el}}^{V}$ is the Coulomb scattering amplitude as given in the Appendix. This form resembles the plane-wave result quite closely: a Coulomb scattering factor ( $1 / q^{2}$ for plane-wave incidence, assuming the form of $f_{\mathrm{el}}^{V}$ here) multiplied by a matrix element $\widetilde{\mathcal{M}}_{f i}$ over the two bound states involved. This matrix element will take on a simpler form below, although the functional form of the "matrix element operator" is not the same as in the plane-wave case, i.e., it is not simply $e^{i \boldsymbol{q} \cdot \boldsymbol{r}}$.

The outgoing plane wave can be written as a series of Bessel functions using the Jacobi-Anger theorem (see also Fig. 4),

$$
e^{-i k_{\perp}^{\prime} \cdot r_{\perp}}=\sum_{\lambda=-\infty}^{+\infty}(-i)^{\lambda} J_{\lambda}\left(k_{\perp}^{\prime} r_{\perp}\right) e^{i \lambda\left(\phi^{\prime}-\varphi\right)}
$$

which makes the remaining hidden azimuthal angular dependence explicit, exposing the possibility of selection rules on (projected) OAM quantum numbers. The matrix element then becomes

$$
\widetilde{\mathcal{M}}_{f i}=\sum_{\lambda=-\infty}^{+\infty}\left(-i e^{i \phi^{\prime}}\right)^{\lambda}\left\langle f\left|J_{\mu}\left(k_{\perp} r_{\perp}\right) J_{\lambda}\left(k_{\perp}^{\prime} r_{\perp}\right) e^{i q_{z} z} e^{i \varphi(\mu-\lambda)}\right| i\right\rangle .
$$

Assuming that the initial and final states are eigenstates of the projected OAM operator $L_{z}$ [i.e., they have the form of Eq. (2)], the azimuthal factor can be integrated out:

$$
\begin{aligned}
\widetilde{\mathcal{M}}_{f i}= & \sum_{\lambda=-\infty}^{+\infty}\left(-i e^{i \phi^{\prime}}\right)^{\lambda}\left\langle\beta\left|J_{\mu}\left(k_{\perp} r_{\perp}\right) J_{\lambda}\left(k_{\perp}^{\prime} r_{\perp}\right) e^{i q_{z} z}\right| \alpha\right\rangle \\
& \times \int \mathrm{d} \varphi \frac{e^{i \varphi\left(m-m^{\prime}\right)}}{2 \pi} e^{i \varphi(\mu-\lambda)} \\
= & \left(-i e^{i \phi^{\prime}}\right)^{\mu+\Delta m}\left\langle\beta\left|J_{\mu}\left(k_{\perp} r_{\perp}\right) J_{\mu+\Delta m}\left(k_{\perp}^{\prime} r_{\perp}\right) e^{i q_{z} z}\right| \alpha\right\rangle \\
= & (-i)^{\mu+\Delta m} e^{i \phi^{\prime}(\mu+\Delta m)} \mathcal{M}_{\beta \alpha}\left(\boldsymbol{k}^{\prime}, \boldsymbol{k}, \mu, \Delta m\right) .
\end{aligned}
$$

The change in the atomic electron's (projected) OAM, $\Delta m=$ $m-m^{\prime}$, is the OAM transferred by the scattering electron in 
the collision. The reduced matrix element is equal to

$$
\mathcal{M}_{\beta \alpha}=\left\langle\beta\left|J_{\mu}\left(k_{\perp} r_{\perp}\right) J_{\mu+\Delta m}\left(k_{\perp}^{\prime} r_{\perp}\right) e^{i q_{z} z}\right| \alpha\right\rangle .
$$

This is now the only unknown. Note that due to the form of $f_{\mathrm{el}}^{V}$ (see the Appendix) and $\widetilde{\mathcal{M}}_{f i}$ (see above), the scattering amplitude's (i.e., the outgoing wave's) azimuthal dependence is exactly $e^{i(\ell+\Delta m) \phi^{\prime}}$, which implies the transfer of the scattering electron's OAM to the atomic state.

For the hydrogen wave functions (oriented with respect to the beam axis, and not the $\theta$-dependent momentum transfer $\boldsymbol{q}$ as in Sec. II A 4), Eq. (30) becomes a triple Bessel integral which can be formally solved using a collection of tricks. The result is, unfortunately, unwieldy and gives no further insight into the physics of the problem.

The next section provides an alternative analytical treatment that can be used to calculate the scattering amplitude for any specific transition, both numerically and analytically. This result is still useful though, when analyzing central scattering, i.e., for $\theta=0$, Eq. (30) simplifies significantly, and due to the generality of the obtained expression, an OAM reciprocity theorem can be deduced. These cases are discussed in Secs. IV B and IV A.

\section{Fourier representation}

The Fourier representation of a Bessel beam given by Eq. (21) provides a solution for the problem of the relative coordinate described in Sec. III A. Starting from Eq. (7) (with $N=(2 \pi)^{5 / 2}$ as in Ref. [27]) and using Eqs. (1) and (21), one arrives at the integral in question ( $\ell^{\prime}$ is the outgoing beam's OAM, obscured by $f_{f i}^{\mathrm{PW}}$ and the integral over the azimuthal Fourier coordinate),

$$
\begin{aligned}
f_{f i}^{\mathrm{V}}\left(\boldsymbol{k}^{\prime}, \ell^{\prime} ; \boldsymbol{k}, \ell\right) & =\frac{(-i)^{\ell}}{2 \pi} \int \mathrm{d} \phi e^{i \ell \phi} f_{f i}^{\mathrm{PW}}(\boldsymbol{q}) \\
& =-\frac{m_{e} e^{2}}{\pi \hbar^{2}}(-i)^{\ell} \int \mathrm{d} \phi \frac{e^{i \ell \phi}}{q^{2}}\left\langle f\left|e^{i \boldsymbol{q} \cdot \boldsymbol{r}}-Z\right| i\right\rangle,
\end{aligned}
$$

where the plane-wave scattering amplitude $f_{f i}^{\mathrm{PW}}(\boldsymbol{q})$ is given by Eq. (11). This can be analytically calculated using the contour integration technique first described in Ref. [27]. Several descriptive examples are treated explicitly below.

Equation (31) has a direct physical interpretation, which is illustrated in Fig. 5. For a certain scattering angle $\theta$ (with respect to the beam's principal direction) and energy $E^{\prime}=$ $\hbar^{2} k^{\prime 2} /\left(2 m_{e}\right)$, each component plane wave of the incoming Bessel beam will coherently interfere and contribute to the outgoing wave. This makes it impossible to define a single momentum transfer $\boldsymbol{q}$ and thus also a unique final-state orientation as discussed in Sec. II A 4. So, in general, a $\phi$-dependent projection is required if one wants to discriminate final states defined with respect to the beam's axis.

To calculate the Bessel-beam scattering amplitude analytically from Eq. (31), one must first obtain Eq. (11) and then apply the following substitutions:

$$
\begin{aligned}
q^{2} & =k_{\perp}^{2}+k_{\perp}^{\prime 2}+q_{z}^{2}-2 k_{\perp} k_{\perp}^{\prime} \cos \left(\phi-\phi^{\prime}\right), \\
z & =\tan \left(\frac{\phi-\phi^{\prime}}{2}\right)
\end{aligned}
$$

$$
\begin{aligned}
\cos \left(\phi-\phi^{\prime}\right) & =\frac{1-z^{2}}{1+z^{2}}, \\
e^{i \ell\left(\phi-\phi^{\prime}\right)} & =\left(\frac{i-z}{i+z}\right)^{\ell}, \\
\mathrm{d}\left(\phi-\phi^{\prime}\right) & =\frac{2 \mathrm{~d} z}{1+z^{2}} .
\end{aligned}
$$

One can then extend the integration over $z$ (over the real axis) to an appropriate contour (such as an infinite semicircle as used for elastic Coulomb scattering [27]) and unleash the residue theorem. As such, the $1 s \rightarrow 1 s$ (elastic) scattering amplitude is

$$
\begin{aligned}
f_{1 s \rightarrow 1 s}^{\mathrm{V}}\left(\boldsymbol{k}^{\prime}, \ell ; \boldsymbol{k}, \ell\right)= & -\frac{2 m_{e} e^{2}}{\hbar^{2}}(-i)^{\ell} e^{i \ell \phi^{\prime}}\left(\frac{R_{2}-R_{1}}{R_{1}+R_{2}}\right)^{|\ell|} \\
& \times \frac{a_{0}^{2} R_{1}^{2} R_{2}^{2}+2 Z^{2}\left(R_{1}^{2}+R_{2}^{2}+4|\ell| R_{1} R_{2}\right)}{a_{0}^{4} R_{1}^{3} R_{2}^{3}}, \\
R_{1}^{2}= & \left(\frac{2 Z}{a_{0}}\right)^{2}+q_{z}^{2}+\left(k_{\perp}-k_{\perp}^{\prime}\right)^{2}, \\
R_{2}^{2}= & \left(\frac{2 Z}{a_{0}}\right)^{2}+q_{z}^{2}+\left(k_{\perp}+k_{\perp}^{\prime}\right)^{2} .
\end{aligned}
$$

Note that the outgoing OAM, $\ell^{\prime}$, is equal to the incoming OAM because none was transferred to the atomic electron. Inelastic scattering amplitudes can also be calculated; below is the result for the $1 s \rightarrow 2 s$ transition:

$$
\begin{aligned}
& f_{1 s \rightarrow 2 s}^{\mathrm{V}}\left(\boldsymbol{k}^{\prime} ; \boldsymbol{k}, \ell\right) \\
&=-\frac{\sqrt{2} m_{e} e^{2}}{\hbar^{2}}\left(\frac{Z}{a_{0}}\right)^{4}(-i)^{\ell} e^{i \ell \phi^{\prime}}\left(\frac{R_{1}-R_{2}}{R_{1}+R_{2}}\right)^{|\ell|} \\
& \times \frac{3\left(R_{1}^{4}+R_{2}^{4}\right)+6|\ell| R_{1} R_{2}\left(R_{1}^{2}+R_{2}^{2}\right)+2\left(1+2 \ell^{2}\right) R_{1}^{2} R_{2}^{2}}{R_{1}^{5} R_{2}^{5}}, \\
& R_{1}^{2}=\left(\frac{3 Z}{2 a_{0}}\right)^{2}+q_{z}^{2}+\left(k_{\perp}-k_{\perp}^{\prime}\right)^{2} \\
& R_{2}^{2}=\left(\frac{3 Z}{2 a_{0}}\right)^{2}+q_{z}^{2}+\left(k_{\perp}+k_{\perp}^{\prime}\right)^{2} .
\end{aligned}
$$

For nonspherical transitions (i.e., where $\Delta m \neq 0$ ), care must be taken about the final-state orientation as discussed in Sec. II A 4. Because there is now no unique momentum transfer $\boldsymbol{q}$, and there is both incoming and outgoing transverse momentum, the rotation over $\phi^{\prime}$ becomes one over $\phi_{q}$ as shown in Figs. 5 and 6 . As an example, we treat the $1 s \rightarrow 2 p$ transitions, for which the plane-wave scattering amplitude is given by Eq. (12). For the rotation over $\phi_{\boldsymbol{q}}$, one can employ the complex representation of vector addition:

$$
q_{\perp} e^{i \phi_{q}}=k_{\perp} e^{i \phi}-k_{\perp}^{\prime} e^{i \phi^{\prime}}=e^{i \phi^{\prime}}\left(k_{\perp} e^{i\left(\phi-\phi^{\prime}\right)}-k_{\perp}^{\prime}\right) .
$$

This, together with Eq. (17b), makes it clear that the integrand in Eq. (31) is dependent only on $\phi-\phi^{\prime}$ and $q$ [which itself is only a function of that same variable; see Eq. (32a)]. For the 


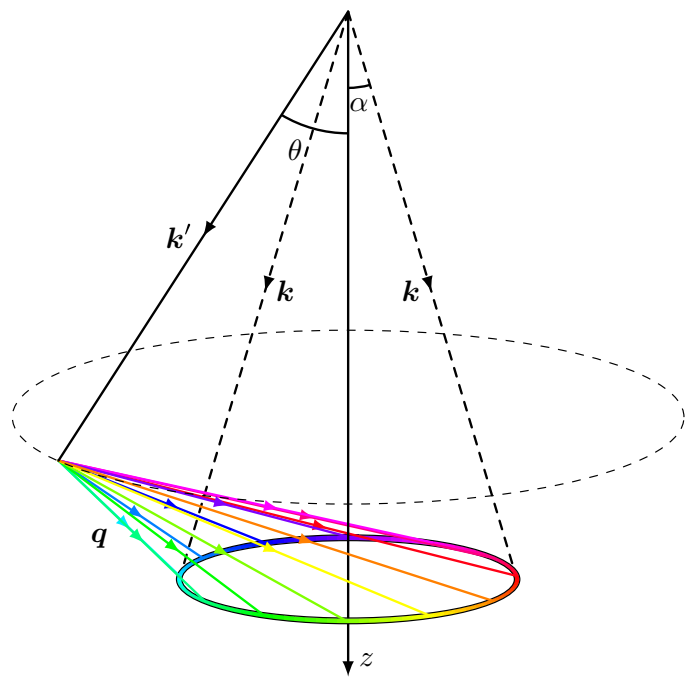

FIG. 5. (Color online) For cylindrical waves, and, by extension, vortex waves, there is no single momentum transfer. Instead, the various plane-wave components of the cylindrical wave contribute to the final outgoing wave-vector component. This is made explicit by the integral over the various momentum component vectors in Eq. (31). A Bessel beam of $\ell=1$ is shown, and the relative phase of the various contributions is shown by the color of the arrows.

$1 s \rightarrow 2 p_{ \pm}$transition, this gives

$$
\begin{aligned}
f_{1 s \rightarrow 2 p_{ \pm}}^{\mathrm{V}}= & -\frac{6 i m_{e} e^{2}(-1)^{\ell}}{\pi \hbar^{2}}\left(\frac{Z}{a_{0}}\right)^{5} e^{i(\ell \mp 1) \phi^{\prime}} \\
& \times \int \mathrm{d}\left(\phi-\phi^{\prime}\right) e^{i \ell\left(\phi-\phi^{\prime}\right)} \frac{k_{\perp} e^{\mp i\left(\phi-\phi^{\prime}\right)}-k_{\perp}^{\prime}}{q^{2}\left[q^{2}+\left(\frac{3 Z}{2 a_{0}}\right)^{2}\right]^{3}}
\end{aligned}
$$

Note that the outgoing wave has lost or gained OAM: the beam electron transfers OAM to the atomic state. The remaining $2 p_{z}$

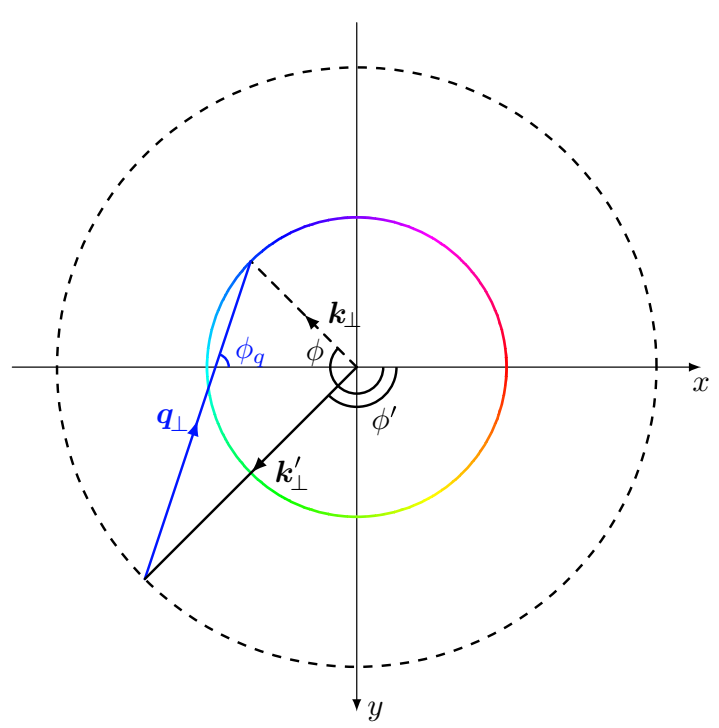

FIG. 6. (Color online) Top-down view of Fig. 5, showing the transverse-plane scattering kinematics for an $\ell=1$ Bessel beam. state has the following scattering amplitude:

$$
\begin{aligned}
f_{1 s \rightarrow 2 p_{z}}^{\mathrm{V}=} & -\frac{6 i \sqrt{2} m_{e} e^{2}(-1)^{\ell}}{\pi \hbar^{2}}\left(\frac{Z}{a_{0}}\right)^{5} q_{z} e^{i \ell \phi^{\prime}} \\
& \times \int \mathrm{d}\left(\phi-\phi^{\prime}\right) e^{i \ell\left(\phi-\phi^{\prime}\right)} \frac{1}{q^{2}\left[q^{2}+\left(\frac{3 Z}{2 a_{0}}\right)^{2}\right]^{3}} .
\end{aligned}
$$

The analytical expressions for these scattering amplitudes can be explicitly written down but are too complex to present here and do not provide much physical insight.

As an alternative to analytical calculation, Eq. (31) can be calculated numerically as a function of the scattering angle by using Eqs. (8) and (17b) and the substitutions

$$
\begin{aligned}
& q_{\perp}^{2}=k_{\perp}^{2}+k^{\prime 2} \sin ^{2} \theta-2 k_{\perp} k^{\prime} \sin \theta \cos \left(\phi-\phi^{\prime}\right), \\
& q_{z}^{2}=\left(k_{z}-k^{\prime} \cos \theta\right)^{2}
\end{aligned}
$$

and integrating over $\mathrm{d}\left(\phi-\phi^{\prime}\right)$ numerically. The functions in question are, all in all, well behaved and standard numerical integration methods should have no issues with them. The option of simple numerical integration is very useful if one wants to explore a different basis set of state wave functions, as long as they can be quantized in OAM as in Eq. (2), and their scattering amplitude can be written as a function of $\phi-\phi^{\prime}$ explicitly.

Figures 8, 9, and 10 show the scattering amplitudes for several characteristic transitions and different input beams. In the limit $\ell=0, k_{\perp}=0$, which is represented by the longdashed (red) line in each of the plots, the results in Fig. 1(b) are recovered. The cross sections are discussed in more detail in Sec. IV C.

\section{DISCUSSION}

\section{A. OAM reciprocity}

In the cylindrical scattering amplitude, Eq. (26), one can consider two specific cases: incoming and outgoing nonvortex states (Fig. 7). For a fixed transition with a certain $\Delta m$, an incoming plane wave (so with $\ell=0, k_{\perp}=0$ ) will result in a scattered vortex wave with $\ell^{\prime}=\Delta m$. Additionally, the summation in Eq. (26) can be performed using $J_{\mu}\left(k_{\perp} r_{\perp}\right) \stackrel{k_{\perp} \rightarrow 0}{\longrightarrow}$ $\delta_{\mu, 0}$. Using the explicit form of $f_{\mathrm{el}}^{V}$ (see the Appendix), one obtains the relatively simple expression

$$
\begin{aligned}
f_{f i}^{\mathrm{PW}}\left(\boldsymbol{k}^{\prime}, \ell^{\prime} ; k_{z} \boldsymbol{e}_{z}\right)= & -\frac{2 m_{e}}{\hbar^{2}}(-i)^{\Delta m} e^{-i \phi^{\prime} \Delta m} \frac{Z e^{2}}{k_{\perp}^{2}+q_{z}^{2}} \\
& \times\left\langle\beta\left|J_{\Delta m}\left(k_{\perp}^{\prime} r_{\perp}\right) e^{i q_{z} z}\right| \alpha\right\rangle .
\end{aligned}
$$

Doing the same, but taking the outgoing wave to be a plane wave $\left(\ell^{\prime}=0, k_{\perp}^{\prime}=0\right)$, one obtains a very similar expression:

$$
\begin{aligned}
f_{f i}^{\mathrm{V}}(\theta=0 ; \boldsymbol{k}, \ell)= & -\frac{2 m_{e}}{\hbar^{2}} \frac{Z e^{2}}{k_{\perp}^{2}+q_{z}^{2}} \delta_{\ell, \Delta m} \\
& \times\left\langle\beta\left|J_{\Delta m}\left(k_{\perp} r_{\perp}\right) e^{i q_{z} z}\right| \alpha\right\rangle .
\end{aligned}
$$

What these two equations say is quite simple: an incident plane wave can gain or lose OAM by exciting a transition and will then be scattered to a certain angle, $\theta=\tan ^{-1}\left(k_{\perp}^{\prime} / k_{z}^{\prime}\right)$. However, a vortex wave with transverse $k_{\perp}$ will have the exact 


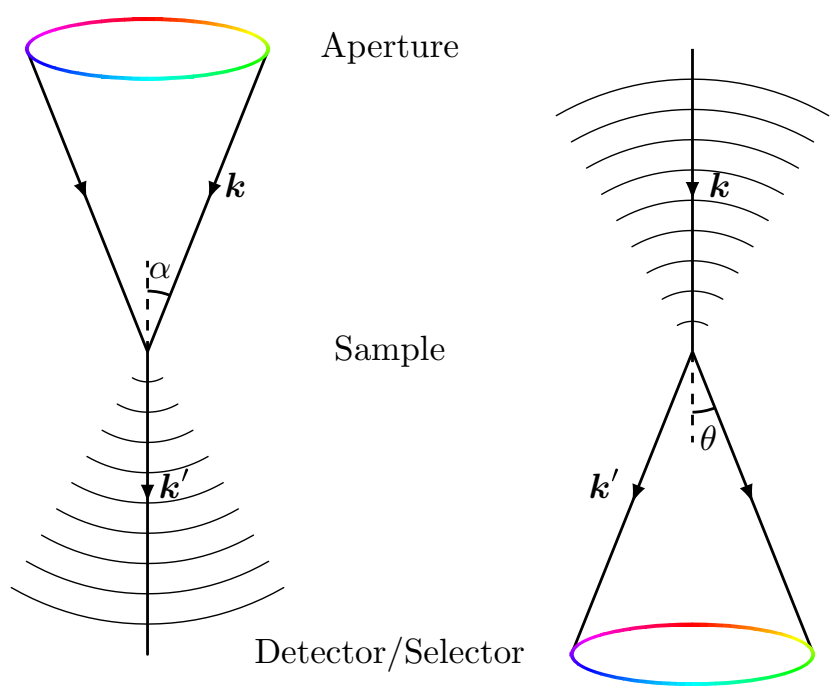

FIG. 7. (Color online) Representation of the symmetry exposed in Sec. IV A between incoming and outgoing vortex waves in the case of incoming and outgoing plane waves, respectively. A specific transition has the same scattering amplitude at the scattering angle $\theta$ corresponding to the inverted geometry as shown here.

same probability of exciting that transition when scattering to $\theta=0$. Note also that only when an incoming vortex beam's OAM matches the transition's $\Delta m$ can it be scattered to $\theta=0$ (see Sec. IV B). This can be seen as a form of reciprocity [40-42], but in this case for inelastic scattering including OAM.

\section{B. Central scattering amplitudes}

Instead of considering the full $\theta$-dependent scattering amplitude, one can also consider only the $\theta=0$ case, for which the analytical expressions are much more tractable than for the complete calculation. Equation (40) can be calculated directly using known integrals [43] of a set of Bessel $K$ and $J$ functions. Alternatively, the analytical method from Sec. III C can be used to the same effect.

For $1 s \rightarrow 1 s$, this gives

$$
\begin{aligned}
& f_{1 s \rightarrow 1 s}^{\mathrm{V}}(\theta=0 ; \boldsymbol{k}, \ell) \\
& \quad=\frac{2 a_{0} e^{2} m_{e} \delta_{\ell, 0}}{\frac{4 Z^{2}}{a_{0}^{2}}+k_{\perp}^{2}+q_{z}^{2}}\left(1+\frac{\left(\frac{2 Z}{a_{0}}\right)^{2}}{\left(\frac{2 Z}{a_{0}}\right)^{2}+k_{\perp}^{2}+q_{z}^{2}}\right) .
\end{aligned}
$$

Note the extra term, which comes from the first term in Eq. (7). If one compares this with the plane-wave result in Ref. [44], one immediately sees the symmetry of this expression if one replaces $k_{\perp}$ with $k_{\perp}^{\prime}$ and realizes $q^{2}=k_{\perp}^{\prime 2}+q_{z}^{2}$ in this situation. This result can also be obtained by setting $k_{\perp}^{\prime}=0$ in Eq. (33).

For the $1 s \rightarrow 2 s$ transition, the following result is obtained:

$$
f_{1 s \rightarrow 2 s}^{\mathrm{V}}(\theta=0 ; \boldsymbol{k}, \ell)=\frac{8 \sqrt{2} m_{e} e^{2}}{\hbar^{2}}\left(\frac{Z}{a_{0}}\right)^{4} \frac{\delta_{\ell, 0}}{\left[k_{\perp}^{2}+q_{z}^{2}+\left(\frac{3 Z}{2 a_{0}}\right)^{2}\right]} .
$$

The Kronecker $\delta$ ensures that the outgoing wave does not gain or lose OAM with respect to the incoming one, as only the $\ell=0$ mode can be nonzero at $\theta=0$.

By considering only central scattering, one can selectively measure a specific transition by preselecting a proper incoming vortex state. This can be shown by considering an incoming $\ell= \pm 1$ vortex beam and $1 s \rightarrow 2 p_{ \pm}$atomic transitions. The relevant central scattering amplitude is given by

$$
\begin{aligned}
& f_{1 s \rightarrow 2 p_{ \pm}}^{V}(\theta=0 ; \boldsymbol{k}, \ell) \\
& \quad=-\frac{12 i m_{e} e^{2}}{\hbar^{2}} \frac{\delta_{\ell, \pm 1}}{k_{\perp}^{2}+q_{z}^{2}}\left(\frac{Z}{a_{0}}\right)^{5} \frac{k_{\perp}}{\left[k_{\perp}^{2}+q_{z}^{2}+\left(\frac{3 Z}{2 a_{0}}\right)^{2}\right]^{3}} .
\end{aligned}
$$

For comparison, the central scattering amplitude for the $1 s \rightarrow$ $2 p_{z}$ transition is

$$
\begin{aligned}
& f_{1 s \rightarrow 2 p_{z}}^{V}(\theta=0 ; \boldsymbol{k}, \ell) \\
& \quad=\frac{12 i \sqrt{2} m_{e} e^{2}}{\hbar^{2}} \frac{\delta_{\ell, 0}}{k_{\perp}^{2}+q_{z}^{2}}\left(\frac{Z}{a_{0}}\right)^{5} \frac{q_{z}}{\left[k_{\perp}^{2}+q_{z}^{2}+\left(\frac{3 Z}{2 a_{0}}\right)^{2}\right]^{3}} .
\end{aligned}
$$

Note that the roles of $k_{\perp}$ and $q_{z}$ are reversed with respect to Eq. (43) (typical behavior for these $p$-character final states) and that the central $p_{z}$ scattering cross section is twice as large for the same parameters. Take special note of the strict selection rule for $\theta=0$ expressed by the Kronecker $\delta$ 's, showing that the on-axis intensity for these transitions will be nonzero only for the right incoming beam. Due to the summation in the full expression, Eq. (26), a mixture of outgoing vortex waves will generally be emitted from each scattering event regardless of its $\Delta m$. The rate at which these various components contribute is determined by the weighting expressed by that equation, which is not trivial.

Finally, it is important to note that the three $2 p$ states considered here are degenerate unless a magnetic field is applied, which adds a Zeeman energy, splitting the nonzero OAM levels with the magnetic field. Larger fields will also induce spin-orbit coupling in an atomic system, further complicating the wave functions and interactions involved $[45,46]$.

\section{Hydrogen scattering amplitudes}

Several properties of the scattering amplitudes presented in Sec. III C, Eqs. (33)-(37), are immediately apparent by looking only at the equations themselves:

(i) Higher energy levels (i.e., larger $n$ 's) introduce higher order $\ell$ dependence.

(ii) Transitions between spherically symmetric states (for which the scattering amplitude is independent of $\phi$ ) depend only on the magnitude of $\ell$, and not on its sign. This is expected, as everything involved is symmetric with respect to rotations around the $z$ axis. Nonsymmetrical transitions depend strongly on the value of $\ell$.

(iii) The scattering electron wave transfers OAM to the atomic state, and this transfer is reflected in the outgoing wave. 

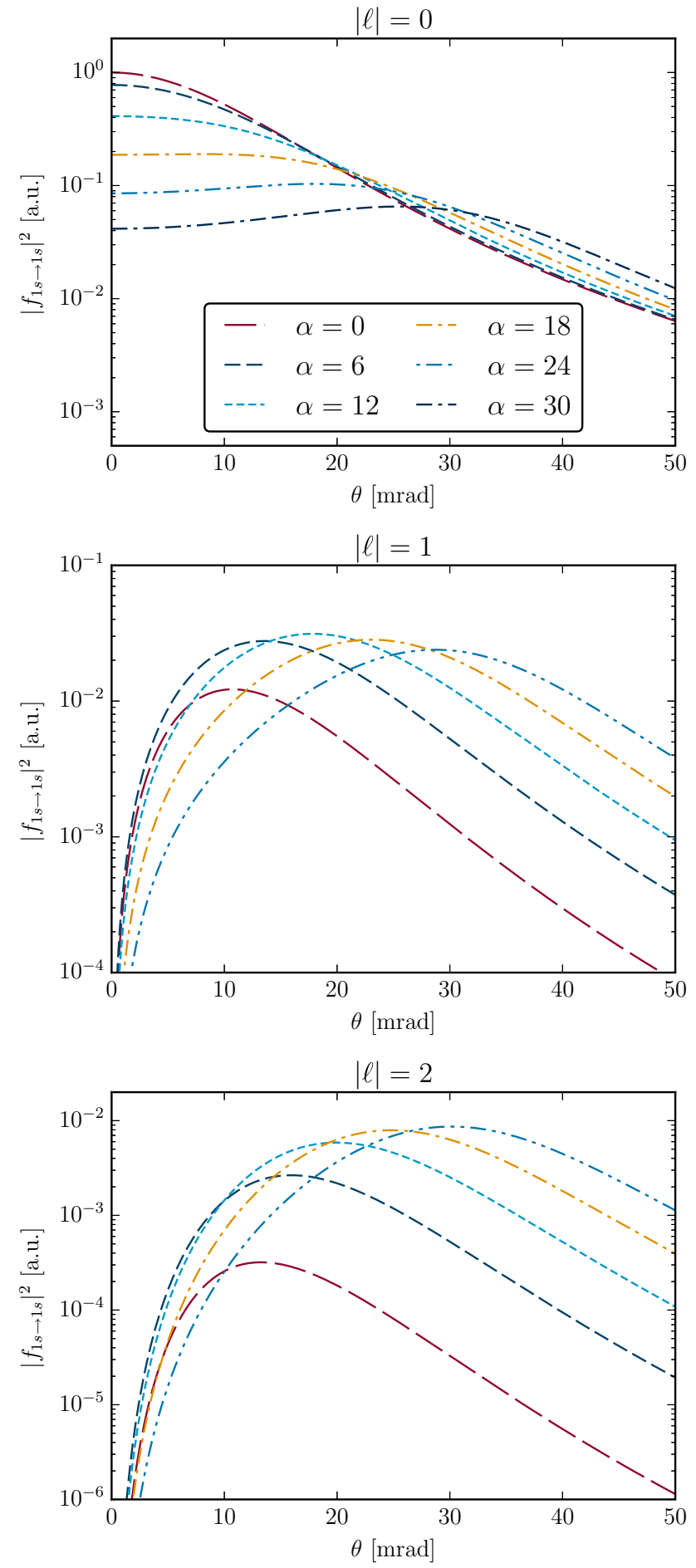

FIG. 8. (Color online) $1 s \rightarrow 1 s$ (elastic) hydrogen differential scattering cross sections for electron Bessel beams of different OAM and transverse momentum, denoted by the Bessel beam opening angle $\alpha$ (in mrad). Similar features as for the screened Coulomb scattering amplitude are present [27].

(iv) Taking the plane-wave limit $\left(\ell=0, k_{\perp} \rightarrow 0\right)$, one recovers the usual plane-wave scattering amplitudes (see $e . g$. Ref. [44] and Fig. 1 b).

The results in Sec. III C are also shown in Figs. 8-10 (in a.u.). The analytical results in Eqs. (33), (34), (36), and (37) and the numerically integrated scattering amplitudes using Eq. (38)
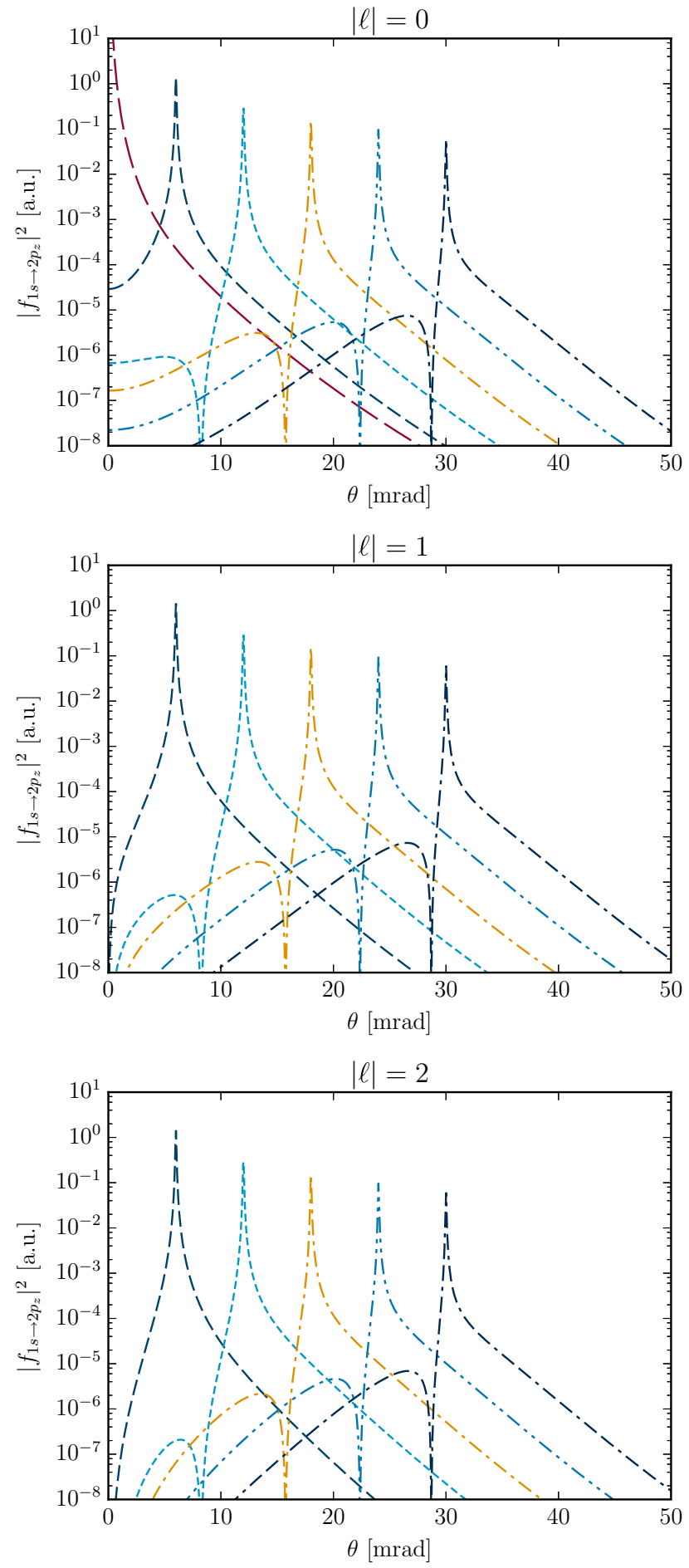

FIG. 9. (Color online) Scattering cross sections for excitation of the $2 p_{z}$ final state. Note that the peaked maximum is smooth when viewed on the much smaller $\theta$ scale of, e.g., Fig. 1(b). The small maximum in the region where $\theta<\alpha$, although real, is relatively insignificant compared to the cross section's maximum value.

were confirmed to be equal. These results and their physical implications are now discussed. Remember that the differential cross section is the probability of an electron being detected at a certain scattering angle, which is the primary interpretation that is given here. Note that the figures show only a line profile; the scattering amplitudes shown here are all cylindrically 

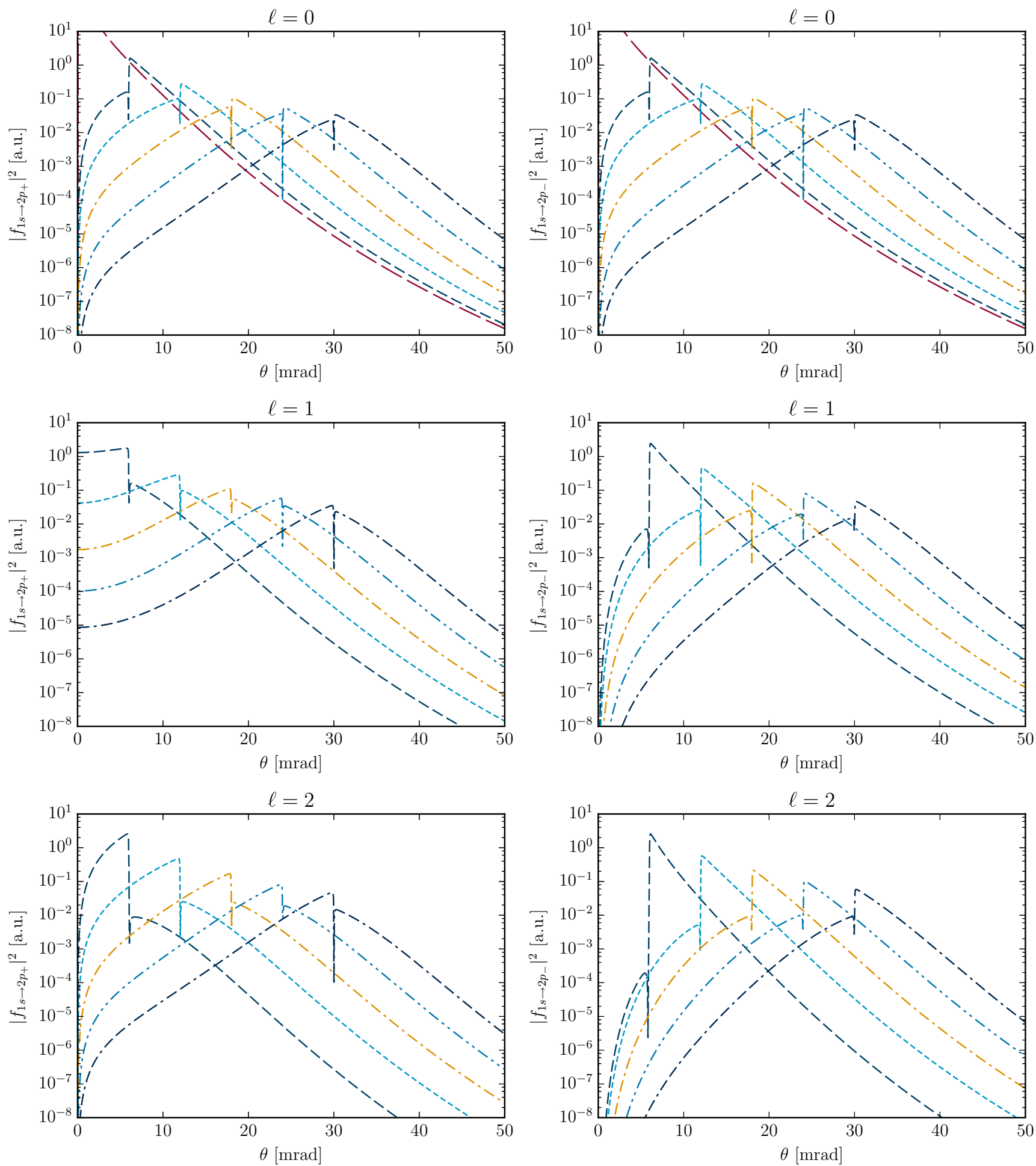

FIG. 10. (Color online) Scattering cross sections for OAM transfer events to the $2 p_{ \pm}$states are shown for a positive incoming beam OAM, $\ell$. Negative $\ell$ results are omitted because they are identical to these with $2 p_{+}$and $2 p_{-}$swapped due to the symmetry between the two situations. The legend is identical to the one in Fig. 8.

symmetrical (up to a possible vortex phase factor), resulting in the scattered electrons forming doughnut-shaped intensity profiles.

The elastic differential scattering cross section, shown in Fig. 8, is very similar to that of the screened Coulomb potential previously treated [27]. For a nonvortex beam, $\ell=0$, higher transverse momentum shifts this peak off-center, although even for an incoming hollow beam (with no low$k_{\perp}$ components, generated by, e.g., an annular aperture), a significant amount of intensity is still scattered on-axis. This can be deduced from the additive contributions of various transverse momentum components to the scattering amplitude 
(shown in the topmost plot in Fig. 8), which additively result in a nonzero on-axis differential scattering cross section. For beams containing a phase vortex, i.e., $\ell \neq 0$, there is no on-axis intensity and the angle at which the cross section peaks shifts outwards with increasing incoming transverse momentum. This feature could be exploited as a sensitive detector for nonOAM-preserving transitions in future experiments. Another spherically symmetric transition is $1 s \rightarrow 2 s$. Its inelastic differential scattering cross section [given explicitly by Eq. (34)] has the same shape and behavior as the $1 s \rightarrow 1 s$ cross section and, therefore, is not explicitly shown.

The differential cross sections for ground-state excitations to various $2 p$ substates (quantized along the beam direction) are shown in Figs. 9 and 10. The $2 p_{z}$ cross section shows a narrow peak that moves away from the beam axis, giving way for a 0 that appears at the threshold transverse momentum defined by the condition $q_{z}=0$ [which is a prefactor in Eq. (37)] and is located at

$$
\theta_{0}=\cos ^{-1}\left(\frac{k}{k^{\prime}} \cos \alpha\right)<\alpha .
$$

When an incoming vortex beam transfers OAM to an atomic electron, the associated differential scattering amplitude reflects this, as shown in Fig. 10. In general, the lower the outgoing OAM is in magnitude $\left|\ell^{\prime}\right|$, the higher the scattering cross section is for low angles. Another general feature of these results is that transitions in which OAM is given to the atomic electron, $\left|\ell^{\prime}\right|<|\ell|$, have a relatively larger differential cross section for scattering angles $\theta<\alpha$. Note that not all OAM needs to be transferred for this to be visible, and these differences are orders of magnitude larger for higher outgoing OAM. Transitions where OAM is taken from the atomic electron, $\left|\ell^{\prime}\right|>|\ell|$, have larger differential cross sections for larger angles, $\theta>\alpha$. This can be clearly seen by comparing the plots in the left and right columns in Fig. 10 (the top row only shows the latter case, $\left.\left|\ell^{\prime}\right|>|\ell|\right)$. Perhaps the most evident form of this is that an $\ell=1$ beam exciting a $1 s \rightarrow 2 p_{+}$transition will scatter most electrons on the beam axis and even to $\theta=0$. This is possible because for this specific transition, the outgoing beam has lost its OAM and does not suffer from the phase singularity previously forcing its amplitude to 0 there. The same happens for an $\ell=-1$ beam exciting a $1 s \rightarrow 2 p_{-}$ transition, as the scattering amplitudes are identical. For higher order incoming beams, this change is less dramatic, as the outgoing vortex phase still forces the central scattering to 0 . Although it is not displayed clearly on the scale used in these figures, the plane-wave differential cross section [long-dashed (red) curve] has the same shape as in Fig. 1(b), and its central 0 gets pushed outwards for higher transverse momentum. The sharp dip at around $\alpha=\theta$ is exactly the 0 of $f_{1 s \rightarrow 2 p_{ \pm}}^{\mathrm{PW}}$ in Fig. 1(b) at $\theta=0$, but shifted outwards due to the nonzero incoming transverse momentum. It coincides with the peak for the $2 p_{z}$ differential cross section at the same scattering angle. These shifts of intensity can be understood intuitively by considering the transverse profiles of a vortex beam, where a higher OAM is generally paired with a larger spatial extent of the wave function. Finally, the large angle scattering in both situations also shows a quantitative offset which could also be used to differentiate the events.

\section{CONCLUSIONS}

We have extended inelastic quantum scattering theory to nontrivial incoming electron waves, including orbital angular and transverse momentum. A quick review of the textbook theory illuminated some often forgotten, but important facts about the final state and momentum transfer. Two methods were then applied to obtain the vortex scattering amplitudes of inelastic transitions for a hydrogen-like system. Special attention was given to the atomic state's OAM and the consequences of it being changed by a scattering electron.

The first method involves the Bessel addition theorem, which leads to unwieldy analytical expressions for all scattering amplitudes. Nonetheless, these calculations led to simple selection rules when OAM transfer was involved and allowed us to estimate the regime of scattering angles for which they are valid. Additionally, a form of OAM reciprocity was shown to exist, tied to the OAM transfer and the central scattering amplitude.

The second approach, using the Fourier representation of the Bessel beam, resulted in a purely analytical method to obtain hydrogen scattering amplitudes, along with a less cumbersome numerical solution using an intermediate result. The scattering amplitudes are influenced strongly in the presence of OAM transfer, even outside of the ideal selection rule validity regime $(\theta=0)$. Combined with energy filtering, the predicted asymmetry could provide a means to better separate scattering contributions for various final states with distinct OAM, leading to an improved measurement of the final-state density. This, in turn, would provide atomic-resolution magnetic information. Specific $\Delta m$ transitions can be filtered from the total scattered intensity using any combination of pre- or postselection of OAM of the scattering electron and incoming and outgoing transverse momentum (i.e., by limiting collection and convergence angles). The selection on transverse momentum is routinely done by choosing detector geometry, selected area apertures, and objective apertures. Postselection on OAM has yet to be practically implemented efficiently in electron microscopes. Once this is in place, though, the use of OAM selectors will significantly improve the selectivity to certain transitions, as a large amount of background signal caused by electrons with the "wrong" OAM will be eradicated.

\section{ACKNOWLEDGMENTS}

Figures were made with TikZ, NumPy and Matplotlib [47-49]. R.V.B. acknowledges support from Fonds Wetenschappelijk Onderzoek-Vlaanderen (FWO) as Aspirant. J.V. acknowledges financial support from the European Union under the Seventh Framework Program (FP7) through a contract for an Integrated Infrastructure Initiative, Reference No. 312483-ESTEEM2, and the European Research Council under the FP7, ERC Starting Grant 278510 VORTEX.

\section{APPENDIX: ELASTIC VORTEX COULOMB SCATTERING AMPLITUDE}

In the cylindrical expansion of the inelastic scattering amplitudes, the elastic scattering amplitude appears. This was calculated in Ref. [27], and the result is repeated 
here:

$$
f_{\mathrm{el}}^{V}\left(\boldsymbol{k}^{\prime} ; \boldsymbol{k}, \ell\right)=-\frac{2 m_{e} V_{0}}{\hbar^{2}} \frac{i^{\ell} e^{i \ell \phi^{\prime}}}{r_{1} r_{2}}\left(\frac{r_{1}-r_{2}}{r_{1}+r_{2}}\right)^{|\ell|}, \quad r_{1}^{2}=q_{z}^{2}+\mu^{2}+\left(k_{\perp}-k_{\perp}^{\prime}\right)^{2}, \quad r_{2}^{2}=q_{z}^{2}+\mu^{2}+\left(k_{\perp}+k_{\perp}^{\prime}\right)^{2} .
$$

Here, $V_{0}$ is either $Z e^{2}$ or $e^{2}$, depending on the context, and the treatment in this paper deals with unscreened Coulomb potentials only, so $\mu=0$ everywhere.

[1] L. Allen, M. W. Beijersbergen, R. J. C. Spreeuw, and J. P. Woerdman, Orbital angular momentum of light and the transformation of Laguerre-Gaussian laser modes, Phys. Rev. A 45, 8185 (1992).

[2] M. R. Dennis, K. O'Holleran, and M. J. Padgett, Singular optics: Optical vortices and polarization singularities, in Progress in Optics, Vol. 53, edited by E. Wolf (Elsevier, Amsterdam, 2009), pp. 293-363.

[3] M. F. Andersen, C. Ryu, P. Cladé, V. Natarajan, A. Vaziri, K. Helmerson, and W. D. Phillips, Quantized rotation of atoms from photons with orbital angular momentum, Phys. Rev. Lett. 97, 170406 (2006).

[4] N. B. Simpson, L. Allen, and M. J. Padgett, Optical tweezers and optical spanners with Laguerre-Gaussian modes, J. Mod. Opt. 43, 2485 (1996).

[5] R. W. Boyd, A. Jha, M. Malik, C. O'Sullivan, B. Rodenburg, and D. J. Gauthier, Quantum key distribution in a high-dimensional state space: Exploiting the transverse degree of freedom of the photon, Proc. SPIE 7948, 79480L (2011).

[6] F. Eilenberger, K. Prater, S. Minardi, R. Geiss, U. Röpke, J. Kobelke, K. Schuster, H. Bartelt, S. Nolte, A. Tünnermann, and T. Pertsch, Observation of discrete, vortex light bullets, Phys. Rev. X 3, 041031 (2013).

[7] O. Matula, A. G. Hayrapetyan, V. G. Serbo, A. Surzhykov, and S. Fritzsche, Atomic ionization of hydrogen-like ions by twisted photons: Angular distribution of emitted electrons, J. Phys. B 46, 205002 (2013).

[8] I. P. Ivanov and V. G. Serbo, Scattering of twisted particles: Extension to wave packets and orbital helicity, Phys. Rev. A 84, 033804 (2011).

[9] I. P. Ivanov, Measuring the phase of the scattering amplitude with vortex beams, Phys. Rev. D 85, 076001 (2012).

[10] P. Schattschneider, M. Stöger-Pollach, S. Löffler, A. SteigerThirsfeld, J. Hell, and J. Verbeeck, Sub-nanometer free electrons with topological charge, Ultramicroscopy 115, 21 (2012).

[11] P. Schattschneider, M. Stöger-Pollach, and J. Verbeeck, Novel vortex generator and mode converter for electron beams, Phys. Rev. Lett. 109, 084801 (2012).

[12] J. Verbeeck, H. Tian, and P. Schattschneider, Production and application of electron vortex beams, Nature 467, 301 (2010).

[13] B. J. McMorran, A. Agrawal, I. M. Anderson, A. A. Herzing, H. J. Lezec, J. J. McClelland, and J. Unguris, Electron vortex beams with high quanta of orbital angular momentum, Science 331, 192 (2011).

[14] L. Clark, A. Béché, G. Guzzinati, A. Lubk, M. Mazilu, R. Van Boxem, and J. Verbeeck, Exploiting lens aberrations to create electron-vortex beams, Phys. Rev. Lett. 111, 064801 (2013).

[15] A. Béché, R. Van Boxem, G. Van Tendeloo, and J. Verbeeck, Magnetic monopole field exposed by electrons, Nature Phys. 10, 26 (2014).
[16] V. Grillo, G. C. Gazzadi, E. Karimi, E. Mafakheri, R. W. Boyd, and S. Frabboni, Highly efficient electron vortex beams generated by nanofabricated phase holograms, Appl. Phys. Lett. 104, 043109 (2014).

[17] O. L. Krivanek, J. Rusz, J.-C. Idrobo, T. J. Lovejoy, and N. Dellby, Toward single mode, atomic size electron vortex beams, Microsc. Microanal. 20, 832 (2014).

[18] K. Yu. Bliokh, Y. P. Bliokh, S. Savel'ev, and F. Nori, Semiclassical dynamics of electron wave packet states with phase vortices, Phys. Rev. Lett. 99, 190404 (2007).

[19] M. Uchida and A. Tonomura, Generation of electron beams carrying orbital angular momentum, Nature 464, 737 (2010).

[20] J. Rusz and S. Bhowmick, Boundaries for efficient use of electron vortex beams to measure magnetic properties, Phys. Rev. Lett. 111, 105504 (2013).

[21] P. Schattschneider, B. Schaffer, I. Ennen, and J. Verbeeck, Mapping spin-polarized transitions with atomic resolution, Phys. Rev. B 85, 134422 (2012).

[22] P. Schattschneider, M. Nelhiebel, and B. Jouffrey, Density matrix of inelastically scattered fast electrons, Phys. Rev. B 59, 10959 (1999).

[23] P. Schattschneider, M. Nelhiebel, H. Souchay, and B. Jouffrey, The physical significance of the mixed dynamic form factor, Micron 31, 333 (2000).

[24] M. Inokuti, Inelastic collisions of fast charged particles with atoms and molecules-The Bethe theory revisited, Rev. Mod. Phys. 43, 297 (1971).

[25] R. F. Egerton and K. Wong, Some practical consequences of the lorentzian angular distribution of inelastic scattering, Ultramicroscopy 59, 169 (1995).

[26] R. F. Egerton, Oscillator-strength parametrization of inner-shell cross sections, Ultramicroscopy 50, 13 (1993).

[27] R. Van Boxem, B. Partoens, and J. Verbeeck, Rutherford scattering of electron vortices, Phys. Rev. A 89, 032715 (2014).

[28] P. Schattschneider, C. Hébert, H. Franco, and B. Jouffrey, Anisotropic relativistic cross sections for inelastic electron scattering, and the magic angle, Phys. Rev. B 72, 045142 (2005).

[29] B. Jouffrey, P. Schattschneider, and C. Hébert, The magic angle: A solved mystery, Ultramicroscopy 102, 61 (2004).

[30] Expanding the exponential in spherical functions separates various contributions to the scattering amplitude into many terms. These were calculated in, e.g., Ref. [50].

[31] L. Calmels and J. Rusz, Momentum-resolved EELS and EMCD spectra from the atomic multiplet theory: Application to magnetite, Ultramicroscopy 110, 1042 (2010).

[32] R. F. Klie, Y. Zhu, G. Schneider, and J. Tafto, Experimental probing of the anisotropy of the empty $p$ states near the Fermi level in $\mathrm{MgB}_{2}$, Appl. Phys. Lett. 82, 4316 (2003). 
[33] G. Berkhout, M. Lavery, J. Courtial, M. Beijersbergen, and M. Padgett, Efficient sorting of orbital angular momentum states of light, Phys. Rev. Lett. 105, 153601 (2010).

[34] K. Saitoh, Y. Hasegawa, K. Hirakawa, N. Tanaka, and M. Uchida, Measuring the orbital angular momentum of electron vortex beams using a forked grating, Phys. Rev. Lett. 111, 074801 (2013).

[35] G. Guzzinati, L. Clark, A. Béché, and J. Verbeeck, Measuring the orbital angular momentum of electron beams, Phys. Rev. A 89, 025803 (2014).

[36] L. Clark, A. Béché, G. Guzzinati, and J. Verbeeck, Quantitative measurement of orbital angular momentum in electron microscopy, Phys. Rev. A 89, 053818 (2014).

[37] Z. Bouchal, J. Wagner, and M. Chlup, Self-reconstruction of a distorted nondiffracting beam, Opt. Commun. 151, 207 (1998).

[38] See, e.g., p. 706 in Ref. [51].

[39] Equation 10.23.7 in Ref. [52].

[40] R. J. Potton, Reciprocity in optics, Rep. Prog. Phys. 67, 717 (2004).

[41] L. Deák and T. Fülöp, Reciprocity in quantum, electromagnetic and other wave scattering, Ann. Phys. 327, 1050 (2012).

[42] S. D. Findlay, P. Schattschneider, and L. J. Allen, Imaging using inelastically scattered electrons in CTEM and STEM geometry, Ultramicroscopy 108, 58 (2007).

[43] Found in Sec. 6.521 of Ref. [53]. Others can be derived by taking the derivative of both sides with respect to the parameters $a$ and $b$ in these equations.
[44] L. I. Schiff, Quantum Mechanics (McGraw-Hill, New York, 1968).

[45] A. Galindo and P. Pascual, Hydrogen atom in a strong magnetic field, Nuovo Cimento B Ser. 34, 155 (1976).

[46] V. S. Popov and B. M. Karnakov, Hydrogen atom in a strong magnetic field, Phys. Uspekhi 57, 257 (2014).

[47] T. Tantau, PGF and TikZ-Graphic systems for TeX; available at: http://sourceforge.net/projects/pgf/ (2013).

[48] S. van der Walt, S. C. Colbert, and G. Varoquaux, The numpy array: A structure for efficient numerical computation, Comput. Sci. Eng. 13, 22 (2011).

[49] J. D. Hunter, Matplotlib: A 2d graphics environment, Comput. Sci. Eng. 9, 90 (2007).

[50] S. Löffler and P. Schattschneider, Transition probability functions for applications of inelastic electron scattering, Micron 43, 971 (2012).

[51] I. N. Bronshtein, K. A. Semendyayev, G. Musiol, and H. Muehlig, Handbook of Mathematics, 4th ed. (Springer-Verlag, New York, 2004).

[52] NIST digital library of mathematical functions, Release 1.0.8 of 2014-04-25 (2014); available at: http://dlmf.nist.gov/ (online companion to [54]).

[53] I. S. Gradshteyn and I. M. Ryzhik, Table of Integrals, Series, and Products (Elsevier, Amsterdam, 2007).

[54] F. W. J. Olver, D. W. Lozier, R. F. Boisvert, and C. W. Clark (eds.), NIST Handbook of Mathematical Functions (Cambridge University Press, New York, 2010) (print companion to [52]). 